\title{
Controlled Gold Nanoparticle Placement into Patterned Polydimethylsiloxane Thin Films via Directed Self-Assembly
}

\author{
Keith R. Berry Jr. (D), ${ }^{1}$ Ricardo L. Romo, ${ }^{2}$ Megan Mitchell, ${ }^{1}$ Vinith Bejugam, ${ }^{3}$ \\ and D. Keith Roper $\mathbb{D i D}^{4}$ \\ ${ }^{1}$ Ralph E. Martin Department of Chemical Engineering, University of Arkansas, Fayetteville, AR 72701, USA \\ ${ }^{2}$ Microelectronics-Photonics Graduate Program, University of Arkansas, Fayetteville, AR 72701, USA \\ ${ }^{3}$ PacTech, Packaging Technologies, Santa Clara, CA 95050, USA \\ ${ }^{4}$ Department of Biological Engineering, Utah State University, Logan, UT 84322, USA
}

Correspondence should be addressed to D. Keith Roper; keith.roper@usu.edu

Received 9 April 2019; Accepted 18 July 2019; Published 5 August 2019

Guest Editor: Laijun Liu

Copyright (c) 2019 Keith R. Berry Jr. et al. This is an open access article distributed under the Creative Commons Attribution License, which permits unrestricted use, distribution, and reproduction in any medium, provided the original work is properly cited.

\begin{abstract}
An economically scalable and reproducible method to assemble nanoparticles (NPs) into ordered arrays with high fidelity remains a fundamental roadblock. Methods like directed self-assembly have shown the highest promise resulting in $>85 \%$ density of NP-filled prepatterned polymer cavities. This work refines directed self-assembly by controlling the evaporation rate, substrate velocity (deposition rate), and NP diameter resulting in reproducible fabrication of ordered arrays with areas $>2 \mathrm{~mm} \times 2 \mathrm{~mm}$ and $\sim 100 \%$ density of filled cavities. Measured optical spectra showed a blueshift in the localized surface plasmon resonance (LSPR) and surface lattice resonance (SLR) peaks with increasing NP density for both 100 and $150 \mathrm{~nm}$ gold (Au) NPs. Discrete dipole approximation (DDA), coupled dipole approximation (CDA), rapid semi-analytical CDA (rsa-CDA), and Mie theory simulations closely matched extinction per nanoparticle (extinction/NP) calculations for measured extinction spectra. An ordered array containing $150 \mathrm{~nm}$ AuNPs was used for comparison with rsa-CDA estimates using both polydimethylsiloxane (PDMS) and glass refractive indices (RI) resulting in peak location estimates within $1.7 \%$ and comparable relative increases in peak heights. Both the measured and simulated SLR peak heights were shown to significantly increase when the array was on glass as opposed to within PDMS.
\end{abstract}

\section{Introduction}

Gold $(\mathrm{Au})$ nanoparticles (NPs) exhibit localized surface plasmon resonance (LSPR) through conduction-electron oscillations when an external electromagnetic (EM) field is applied [1-3]. When the AuNPs are ordered into a square lattice and exposed to an external EM field, the LSPRs on each individual particle couple with light diffracted from the ordered arrangement resulting in a surface lattice resonance (SLR), i.e., a Fano resonance or coupled lattice resonance (CLR), spectral feature corresponding to the lattice spacing [3-10]. SLR features can be tuned by varying the lattice spacing, NP geometry, incident EM field inten- sity, and refractive index (RI) $[8,9,11-16]$. Simulations, such as plasmon hybridization, coupled dipole approximation (CDA), rapid semi-analytical CDA (rsa-CDA), finitedifference time-domain (FDTD), and Fano theory, have been used to estimate both the LSPR and SLR peak locations, peak heights, scattering amplitudes, and scattering cross-sections [5, 7, 10, 17-19].

Popular commercial microfabrication techniques, such as electron beam lithography (EBL), nanoimprint lithography (NIL), laser ablation (LA), and focused ion beam (FIB), have been used to produce random and ordered assemblies of AuNPs [12, 20-24]. Recent advances in the fabrication of ordered arrays include thermal evaporation, dip-coating 
self-assembly, template-assisted self-assembly, and directed self-assembly $[4,19,25-35]$. In particular, a variety of selfassembly techniques have been developed and employed to cost-effectively fabricate arrays of AuNPs. These techniques range from receding contact line, i.e., meniscus moving, approaches (the solution is between a fixed glass superstrate and a fixed substrate where evaporation controls the deposition of AuNPs) to fixed glass superstrates with moving substrates (the solution between a fixed glass superstrate and a substrate attached to a linear translation stage where evaporation and the rate of substrate motion controls the deposition) [26, 27, 30-32]. These advances offer economically scalable approaches to fabricate ordered arrays of AuNPs, but a reproducible approach that can produce high-density, millimeter-scale areas with single AuNP per cavity is lacking.

This work introduced a refined directed self-assembly process for fabricating ordered arrays of AuNPs yielding $>2$ $\mathrm{mm} \times 2 \mathrm{~mm}$ areas of $\sim 100 \%$ density of filled prepatterned polydimethylsiloxane (PDMS) cavities containing one NP per cavity. Extinction spectra and extinction per NP (extinction/NP) values showed blueshifting of the LSPR and SLR peaks as the density of AuNPs increased. Mie theory and DDA closely matched measured values for extinction/NP for LSPR peaks of both 100 and $150 \mathrm{~nm}$ AuNPs. CDA and rsa-CDA resulted in greater differences between measured extinction/NP values but closely matched the measured peak locations for both LSPR and SLR for $150 \mathrm{~nm}$ AuNPs. Discrepancies between these simulated results for CDA and measured results were likely a function of RI because the measured values were surrounded by air, water, Triton X100 , and PDMS while simulated values assumed only PDMS surrounded the AuNPs. An ordered array containing $150 \mathrm{~nm}$ AuNPs was spectroscopically characterized before being transferred onto a glass slide and spectroscopically characterized again. rsa-CDA simulations were performed using PDMS and glass RI and compared to the measured spectra, which showed that peak locations for LSPR, quadrupole, and SLR features matched within $1.7 \%$. Relative increases in peak heights also closely matched for rsa-CDA simulation and measured extinction spectra.

\section{Materials and Methods}

2.1. Fabrication of 2D Array Stamps. Silicon (Si) master stamps lithographed with a square lattice of posts at a $600 \mathrm{~nm}$ pitch (150 nm post height; $195 \mathrm{~nm}$ post diameter) were purchased from LightSmyth Technologies (Eugene, OR, S2D-24B2-0808-150-P). Polydimethylsiloxane (PDMS) monomer and curing agent (Sylgard 184 silicone elastomer kit; \#240 401 9862) were purchased from Dow Corning Corporation (Midland, MI). Monomer and curing agent were mixed at a 10:1 monomer:cross-linker ratio at $3000 \mathrm{rpm}$ for 6 minutes in a speed mixer purchased from FlackTek Inc. (\#DAC 150SP/601 0064, Landrum, SC). The mixture was then degassed until observable air bubbles were removed (5-25 minutes) and poured onto a prerinsed (acetone+water) Si stamp and cured for $15 \mathrm{~min}$ at $180^{\circ} \mathrm{C}$. Cured PDMS templates with $600 \mathrm{~nm}$ cavity arrays were peeled away from the
Si stamp. PDMS arrayed-cavity stamp thickness averaged $300 \mu \mathrm{m}$.

2.2. Solution Preparation. Triton X-100 purchased from Sigma-Aldrich (St. Louis, MO) was diluted to 1 weight (wt) $\%$ in DI water and nitrogen capped (nitrogen gas was blown into the vessel for $\sim 1 \mathrm{~min}$ ) to remove air bubbles. 100 and $150 \mathrm{~nm}$ citrate-stabilized AuNPs (stock: 6 and $6.4 \mathrm{mg} / \mathrm{mL}$, respectively) purchased from Nanopartz (Loveland, CO) were diluted to 3 and $3.2 \mathrm{mg} / \mathrm{mL}$, respectively, in $1 \mathrm{wt} \%$ Triton $\mathrm{X}-100$. The solution was nitrogen capped to remove air bubbles.

2.3. Stage Preparation. A substrate with a cavity (see Scheme 1) was built using glass slides (VWR, 16004-430) to support and translocate the stamp and slow evaporative AuNP deposition. The substrate consisted of a 2 in $\times 3$ in single glass slide base (Premiere, 6101) supporting four glass slide walls (VWR, 16004-430) forming a $4 \mathrm{~mm} \times 20 \mathrm{~mm}$ cavity with a depth of $\sim 1 \mathrm{~mm}$ in the center as shown in Schemes 1(a)-1(d). The walls were one glass slide thick $(1 \mathrm{~mm})$ and were cut using a diamond knife and then attached to the base using epoxy resin (Loctite, heavy duty). The substrate was attached via carbon tape to a motorized syringe pump (KD Scientific, KDS-100) as shown in Scheme 1(c). A fixed superstrate was built by using superglue (Loctite, Super Glue Gel) to attach a $2 \mathrm{~mm} \times 2 \mathrm{~mm}$ glass slide with a thickness of $1 \mathrm{~mm}$ (VWR, 16004-430) that was cut with a diamond knife to a second 1 in $\times 3$ in glass slide for high evaporation experiments (Scheme 1(a)) or a 2 in $\times 3$ in single glass slide for low evaporation experiments (Scheme 1(b)). The purpose of this $2 \mathrm{~mm} \times 2 \mathrm{~mm}$ glass slide was to facilitate contact line pinning of the NP solution on the PDMS arrayed-cavity stamp as shown in Scheme 1(d). The $2 \mathrm{~mm} \times 2 \mathrm{~mm}$ glass slide on the 2 in $\times 3$ in slide for low evaporation experiments was surrounded by a single layer of glass coverslips (Corning, 2845-18, 0.12-0.16 mm thickness) that were spaced $\sim 5 \mathrm{~mm}$ from each side of the $2 \mathrm{~mm} \times 2 \mathrm{~mm}$ slide as shown in Scheme 1(e). The superstrate was then attached to a 3 -axis positioner (Line Tools Company, Model A-LH) so that the $2 \mathrm{~mm} \times 2 \mathrm{~mm}$ glass slide could be lowered into the $4 \mathrm{~mm} \times 20 \mathrm{~mm}$ cavity containing the stamp.

2.4. Gold Nanoparticle Deposition. The PDMS arrayed-cavity stamp was cut in half using a razorblade so that two experiments could be performed with each stamp. A half-stamp was placed inside the substrate cavity, and a $2.5 \mu \mathrm{L}$ drop of 1 wt\% Triton X-100 in DI water was added to the bottom center of the stamp. The superstrate was then lowered into position so that the drop pinned to the $2 \mathrm{~mm} \times 2 \mathrm{~mm}$ glass slide and lowered until it spread and pinned to all four sides of the slide (as shown in Schemes 1(a) and 1(d)). The linear stage was then set to $8.0 \mu \mathrm{m} / \mathrm{s}$. A cardboard box was placed over the system to minimize evaporation due to ambient light and convection. This process acted as a precoat to increase AuNP deposition. Once the precoat was finished ( $15 \mathrm{~min})$, a $2.5 \mu \mathrm{L}$ drop of $3(100 \mathrm{~nm}$ AuNPs) or $3.2 \mathrm{mg} / \mathrm{mL}(150 \mathrm{~nm}$ AuNPs) in $1 \mathrm{wt} \%$ Triton X-100 was placed in the same 


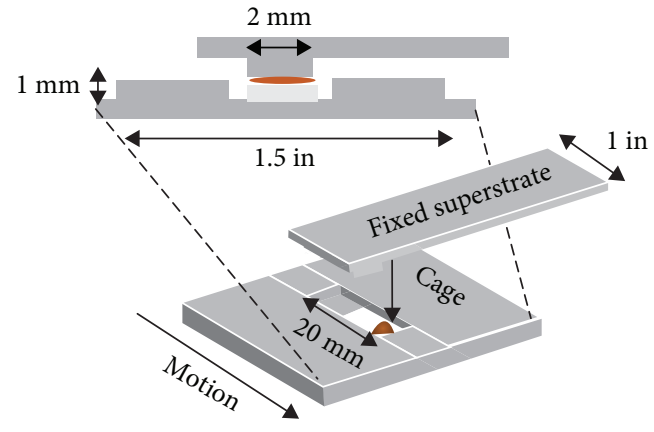

(a)

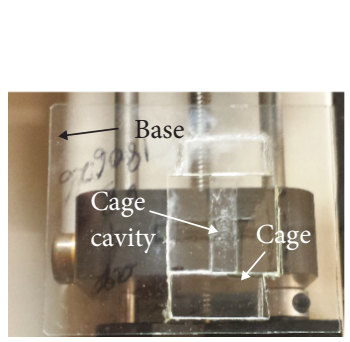

(c)

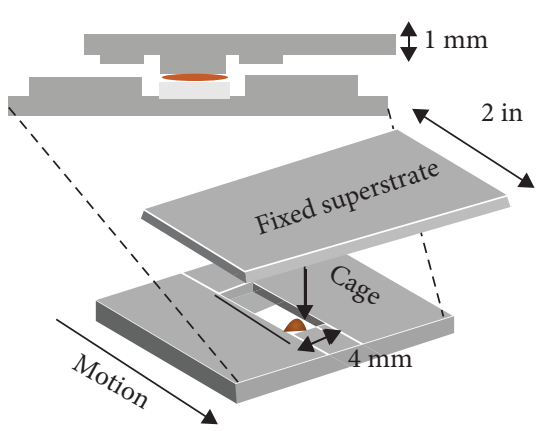

(b)

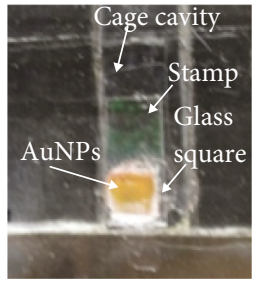

(d)

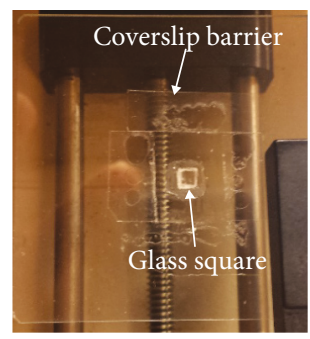

(e)

Scheme 1: Schematic of directed self-assembly stage setup used for (a) high evaporation and (b) low evaporation experiments. (c) Shows the location on the syringe pump where the cage is attached. (d) Shows an example of a drop of AuNPs pinned by the fixed superstrate at the beginning of a high evaporation self-assembly experiment. (e) Shows the superstrate used for the low evaporation self-assembly experiments.

position and the superstrate was lowered until the drop pinned on all four sides of the glass slide. The linear stage was set at rates ranging from $2.1 \mu \mathrm{m} / \mathrm{s}$ to $1.2 \mu \mathrm{m} / \mathrm{s}$.

2.5. Optical Characterization. Transmission spectra of each AuNP ordered array were measured using a light microscope (Eclipse LV100, Nikon Instruments, Melville, New York) integrated with a UV-Vis spectrometer (Shamrock 303, Andor Technology, Belfast, UK). Extinction amplitudes for both LSPR and SLR peaks were taken using a tangent line corresponding to each peak. The extinction was normalized by setting the valley before the SLR peak to 0.01 A.U. for the extinction of each AuNP array. Dark-field microscopic images were captured at $20 \mathrm{x}$ and $100 \mathrm{x}$ objectives using the same light microscope used for the spectral characterization.

\subsection{Rapid Semi-analytical Coupled Dipole Approximation} (rsa-CDA) Simulations. Rapid semi-analytical coupled dipole approximation (rsa-CDA) was performed to determine estimated spectral peak locations for arrays of AuNPs with a diameter of $150 \mathrm{~nm}$ using a previously developed method $[5,36,37]$. Briefly, this configuration treats each AuNP as a single dipole point with a dipole polarizability of, $\alpha$. The NPs were arranged in a $2 \mathrm{D}$ square lattice with an array dimension of $301 \times 301$ and a lattice constant, distance from the center of one NP to the adjacent NP, set at $600 \mathrm{~nm}$. The simulation was performed across a wavelength range of $500-950 \mathrm{~nm}$ for refractive indices (RI) of PDMS (1.42) and glass (1.52). The simulation outputted extinction efficiencies across the spectral range. The primary observable features were the SLR peak, the LSPR peak, and the quadrupole peak (150 nm only).
2.7. Discrete Dipole Approximation (DDA) and CDA Simulations. Discrete dipole approximation (DDA) simulations were performed in a 12-core supercomputer node with a $64 \mathrm{~GB}$ memory for 500 to $950 \mathrm{~nm}$ PDMS (1.42) wavelengths and a resolution of $5 \mathrm{~nm}$. Target and parameter files for DDSCAT 7.3 were generated, using a custom-made MATLAB tool available on nanoHUB [38], for 100 and $150 \mathrm{~nm}$ diameter AuNPs. The generated spectra for a single AuNP were output into Microsoft Excel for further analysis, and real and imaginary parts of the polarizability were stored for ordered array simulations. The polarizability data was then imported into MATLAB for CDA to generate a $301 \times$ 301 array of AuNPs with a lattice spacing of $600 \mathrm{~nm}$ in PDMS (1.42). The generated spectra for both $100 \mathrm{~nm}$ and $150 \mathrm{~nm}$ diameter AuNP arrays were output into Microsoft Excel for further analysis.

2.8. Extinction per NP Calculations. Estimates of extinction per NP were calculated previously using Mie solutions to Maxwell's equations, DDA, and CDA by Dunklin et al. [2]. Briefly, extinction per NP for a single particle Mie theory was determined via $\sigma\left(\log _{10} e\right) / n$, where $\sigma$ was the Mie theory extinction cross-section in $\mathrm{cm}^{2}, \log _{10} e$ was a conversion factor between log bases, and $n$ was the spectrum-averaged RI of PDMS (1.42). DDA and CDA output extinction efficiency were converted to an extinction cross-section before being converted to extinction per NP. The conversion from extinction efficiency to extinction cross-section was performed by multiplying the extinction efficiency by the NP geometric cross-section, i.e., $\pi r^{2}$, where $r$ was the radius of the sphere ( 50 or $75 \mathrm{~nm}$ ). Extinction per NP for the current work was determined by $A / c n$, where $A$ is the extinction peak height 
and $c$ is the density of NPs in a square lattice assuming one particle per cavity with an interparticle spacing of $600 \mathrm{~nm}$.

\section{Results and Discussion}

3.1. Current Self-Assembly Approaches. Possible options to fabricate ordered arrays of AuNPs using patterned substrates include self-assembly techniques like templateassisted self-assembly, dip-coating self-assembly, and directed self-assembly. Template-assisted self-assembly is a process that uses a patterned substrate (e.g., templated PDMS and templated silicon wafer) as a template to facilitate the NPs in solution to arrange in a regular, periodic structure either within the patterned substrate or on a separate substrate [19]. In dip-coating self-assembly, a hydrophilic or hydrophilized patterned substrate is dipped at controllable rates for various time intervals into a solution of NPs, where the NPs are drawn into the patterned cavities via convective and capillary forces $[28,29]$. In directed self-assembly, a patterned substrate is attached to a linear translation stage and a fixed glass slide is used as a superstrate where a droplet of NPs is sandwiched between the fixed superstrate and the patterned substrate that moves at a controllable velocity [26, 27].

Table 1 summarizes the four key features of current selfassembly approaches and the method described in this work: (1) structure and dimensions of the nanostructure, (2) fabrication conditions, (3) substrate on which the nanostructure is fabricated, and (4) fidelity achieved. Matricardi et al. introduced a template-assisted self-assembly method to fabricate ordered arrays of close-packed, mesoscopic clusters of AuNPs onto a hydrophilized glass coverslip by sandwiching a drop of AuNPs between a patterned PDMS substrate and a hydrophilized glass coverslip superstrate [19]. Bejugam introduced an alternative template-assisted self-assembly method that sandwiched a 1-2 $\mu \mathrm{L}$ droplet of AuNPs between a PDMS-PEO copolymer substrate contained within a walled cage and a glass coverslip that sealed the cage and pinned the droplet to three sides of the substrate, after which the droplet was left to evaporate for $>6$ hours [4]. Kinnear et al. introduced a dip-coating self-assembly method using a silanized patterned PDMS substrate, and Juillerat et al. introduced a dip-coating self-assembly method using a hydrophilic patterned PMMA substrate, in which both substrates were dipped into solutions of NPs at various controlled rates $[28,29]$. Cerf and Vieu used a directed self-assembly approach with a suspension of citrate-coated AuNPs containing $1 \mathrm{wt} \%$ Triton X-100 surfactant to increase the deposition in a hydrophobic patterned PDMS substrate, while Fan et al. used a much slower substrate velocity $(0.6 \mu \mathrm{m} / \mathrm{s})$, lower controlled temperature $\left(21^{\circ} \mathrm{C}\right)$, and a controlled contact angle $\left(25^{\circ}\right)$ rather than surfactant or a hydrophilic patterned substrate to deposit silica-gold nanoshells into ordered arrays in PDMS $[26,27]$. Based on the approaches described above, directed self-assembly offers the best approach to achieve high fidelity ordering of single AuNPs per cavity in a PDMS substrate across millimeter scale areas with high reproducibility and scalability.

This work introduces a refined directed self-assembly approach that uses a modified fixed glass superstrate and uti- lizes contact line pinning that conforms the droplet across a small square glass slide for deposition to occur. In conjunction with the modified fixed glass superstrate, the substrate was contained within a walled cage that reduced the evaporation rate of the droplet rather than controlling the temperature. Controlling the evaporation rate with the walled cage and modified superstrate offers a reproducible and scalable method for fabricating ordered arrays under ambient conditions. Varying the substrate velocity (deposition rate), AuNP diameter, and evaporation rate resulted in densities of filled cavities between $85 \%$ and $100 \%$ with single AuNPs per cavity in higher density arrays.

\subsection{Developing Uniform, Large-Area AuNP Ordered Arrays.} The development of a scalable, economic, and reproducible approach to fabricating large-area ordered arrays with high fidelity, density, and accuracy remains important for the advancement of electronic, sensing, and photonic devices. Directed self-assembly, in particular, offers a scalable, economic approach for fabricating ordered arrays with high fidelity and densities ranging from 70 to $85 \%$ for single particles per cavity and $>85 \%$ for multiple particles per cavity [26, 27, 30-32]. This work refines a directed selfassembly approach yielding densities of $\sim 100 \%$ for largearea $(>2 \mathrm{~mm} \times 2 \mathrm{~mm}$ ) arrays by manipulating three primary variables: (1) evaporation rate, (2) deposition rate, and (3) NP diameter. The evaporation rate in this work was primarily due to the head-space within the walled cage when the superstrate was in contact with the droplet of AuNPs, the separation distance between the stamp and the superstrate, the separation distance between the cage and the superstrate, and the overall size of the superstrate that affected the airflow potential. It was observed that increasing the size of the superstrate resulted in a lower evaporation rate and higher deposition. Reducing the deposition rate resulted in higher overall densities for both high and low evaporation conditions. Increasing the AuNP diameter resulted in a more viscous solution with relatively similar concentrations of AuNPs, which also improved the densities while reducing evaporation potential due to the increased viscosity of the solution. These variables were adjusted to fabricate the six arrays shown in Figure 1.

Figure 1 shows the results for six arrays fabricated using two different evaporation rates (high and low), two different AuNP diameters (100 and $150 \mathrm{~nm}$ ), and four different deposition rates $(2.1,1.8,1.5$, and $1.2 \mu \mathrm{m} / \mathrm{s})$. A deposition rate of $1.2 \mu \mathrm{m} / \mathrm{s}$ (lower evaporation rate and $150 \mathrm{~nm}$ AuNP diameter held constant) was performed resulting in a $>2 \mathrm{~mm} \times 2 \mathrm{~mm}$ area of $\sim 100 \%$ filled cavities with high uniformity as shown in the bottom right (red filled triangle) images in Figure 1. The 20x inset shows a uniform color across the entire image while the 100x image shows the high density of filled cavities. Matricardi et al. and Zhang et al. report that the uniform color across the entirety of the array suggests that the nanoparticles are aligned and deposited in a uniform and reproducible manner within each cavity $[19,33]$. The results for the bottom right image (red filled triangle) differ from many of the reported processes in that the array appears to consist of a uniform area of single AuNPs per cavity while reports by 


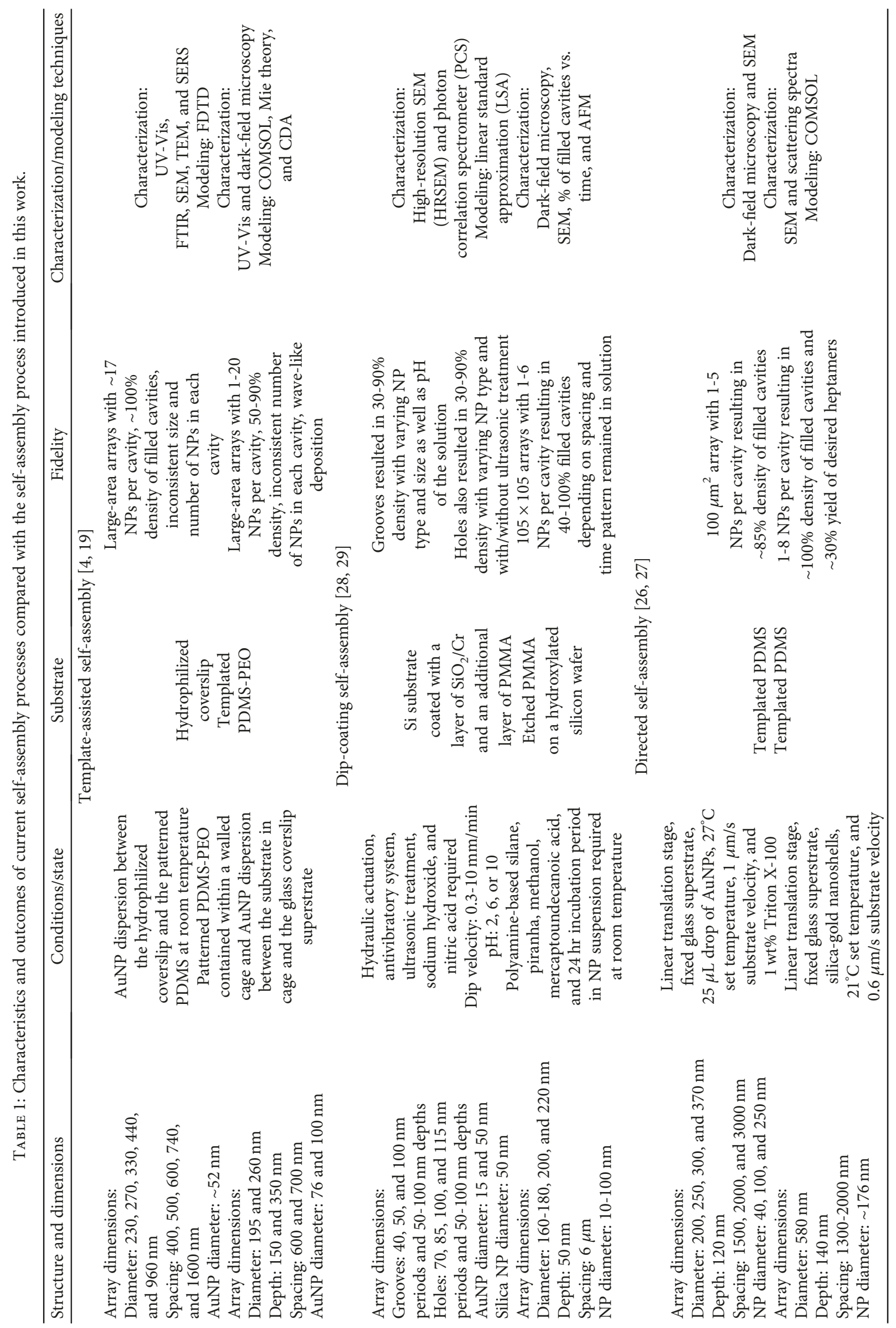




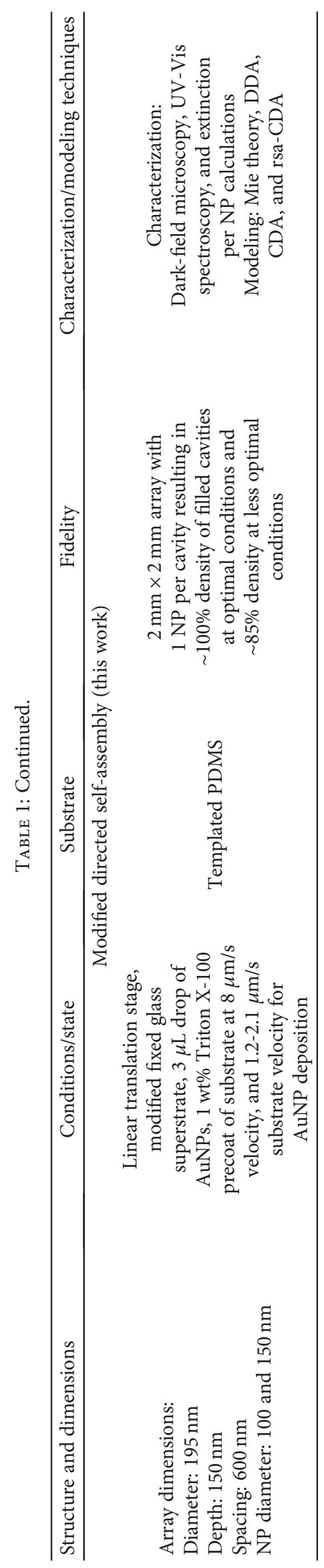




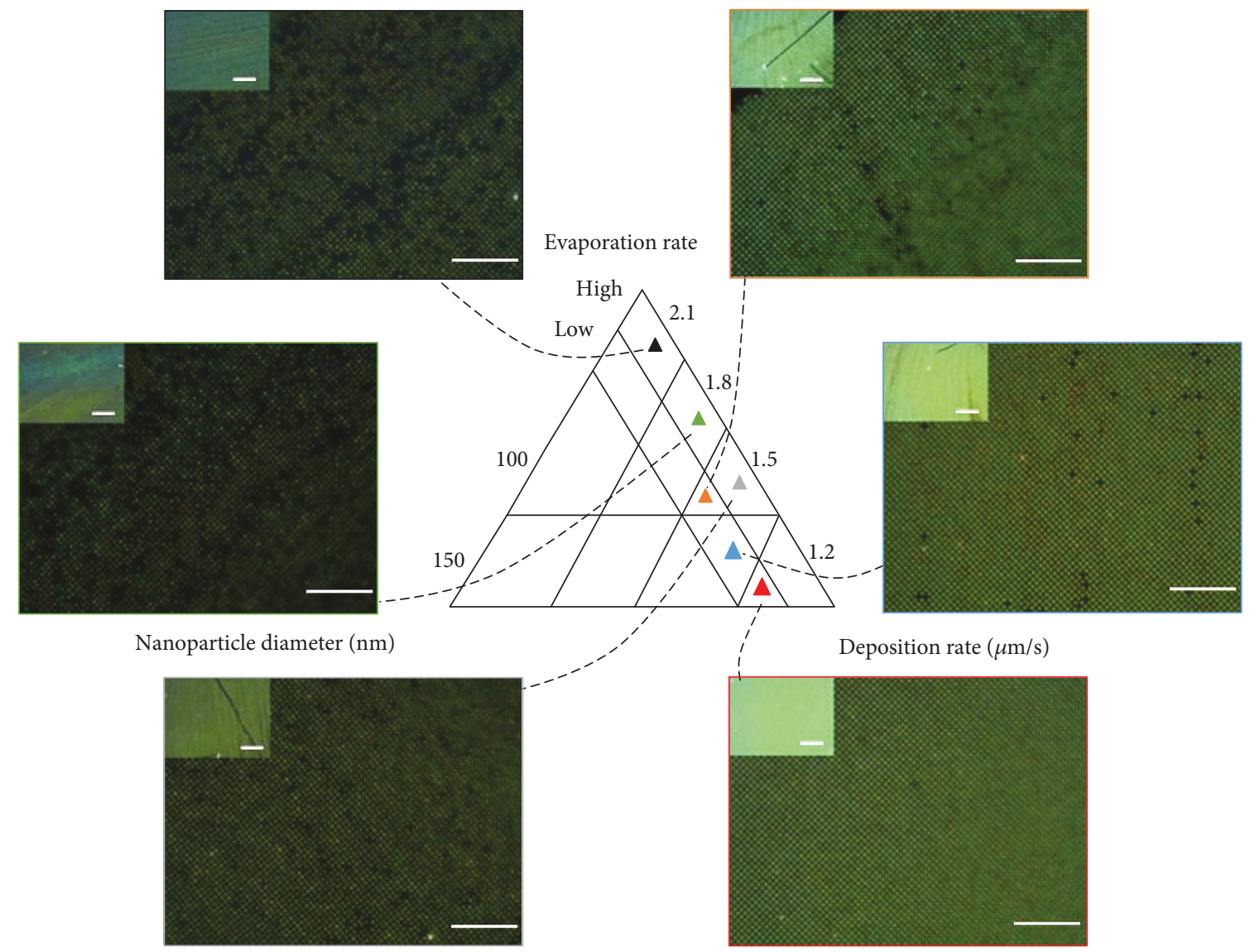

FIGURE 1: Controlled deposition via NP diameter, deposition rate, and evaporation rate resulted in uniform, large-area $(>2 \mathrm{~mm} \times 2 \mathrm{~mm})$ arrays of $\sim 100 \%$ ordering. As the evaporation and deposition rates increased and NP diameter decreased, the uniformity, density, and ordering decreased as shown in 100x images (scale bar: $30 \mu \mathrm{m}$ ) and 20x insets (scale bar: $1000 \mu \mathrm{m}$ ).

Cerf and Vieu, Zhang et al., Matricardi et al., Fan et al., and Juillerat et al. show multiple particles per cavity [19, 26-28, 33]. The AuNP diameter of $150 \mathrm{~nm}$ and the combination of water and Triton X-100 within a cavity with dimensions of $150 \mathrm{~nm} \times 195 \mathrm{~nm}($ depth $\times$ diameter $)$ suggest that only a single AuNP could be within the cavity. Using a slightly faster deposition rate $(1.5 \mu \mathrm{m} / \mathrm{s})$ resulted in slightly lower uniformity and a lower density of filled cavities as shown in the middle right (blue filled triangle) 20x inset and 100x image, respectively. A calculable NP density was determined by counting the number of unfilled cavities and the total number of cavities in the $100 \mathrm{x}$ image, which showed that $98 \%$ of the cavities were filled.

Decreasing the AuNP diameter resulted in lower densities of filled cavities and a higher likelihood of multiple AuNPs per cavity based on lower uniformity in color across the array. The first array (black filled triangle) was fabricated using a high evaporation rate, a deposition rate of $2.1 \mu \mathrm{m} / \mathrm{s}$, and $100 \mathrm{~nm}$ AuNP diameter, which resulted in the 20x image (inset) with observable nonuniform multicolored deposition and the $100 \mathrm{x}$ image that shows $\sim 85 \%$ density of filled cavities. When maintaining the high evaporation rate (smaller superstrate) and $100 \mathrm{~nm}$ AuNP diameter while simultaneously decreasing the deposition rate to 1.8 and $1.5 \mu \mathrm{m} / \mathrm{s}$, the uniformity and density increase resulting in the middle left (green filled triangle) and bottom left (gray filled triangle) images, respectively. The 20x images show the improved uniformity at the lower deposition rate $(1.5 \mu \mathrm{m} / \mathrm{s})$, and the $100 \mathrm{x}$ images show the improved density of filled cavities ( $90 \%)$. Decreasing the deposition rate to $1.2 \mu \mathrm{m} / \mathrm{s}$ while leaving the AuNP diameter and evaporation rate constant resulted in little deposition due to the droplet of AuNPs disconnecting from the glass slide on the superstrate as a result of the high evaporation rate. Using a low evaporation rate (larger superstrate) and maintaining the AuNP diameter $(100 \mathrm{~nm})$ and deposition rate $(1.5 \mu \mathrm{m} / \mathrm{s})$ result in the top right (orange filled triangle) images, which show a small improvement in the uniformity (20x inset) and density (100x image).

3.3. Optical Properties of AuNP Ordered Arrays. The optical responses of ordered arrays of AuNPs generally determine the sensing efficiencies of ordered AuNPs, where the LSPR and SLR peak locations and peak heights account for a majority of the tunable optical responses for sensing applications [19]. Extinction spectra were performed for the arrays shown in Figure 1 and normalized so that the valley just before the SLR feature was set to 0.01 absorbance units (A.U.) and displayed in Figure 2. The array images from Figure 1 were outlined in the corresponding line color for the extinction spectra and arranged to the right 


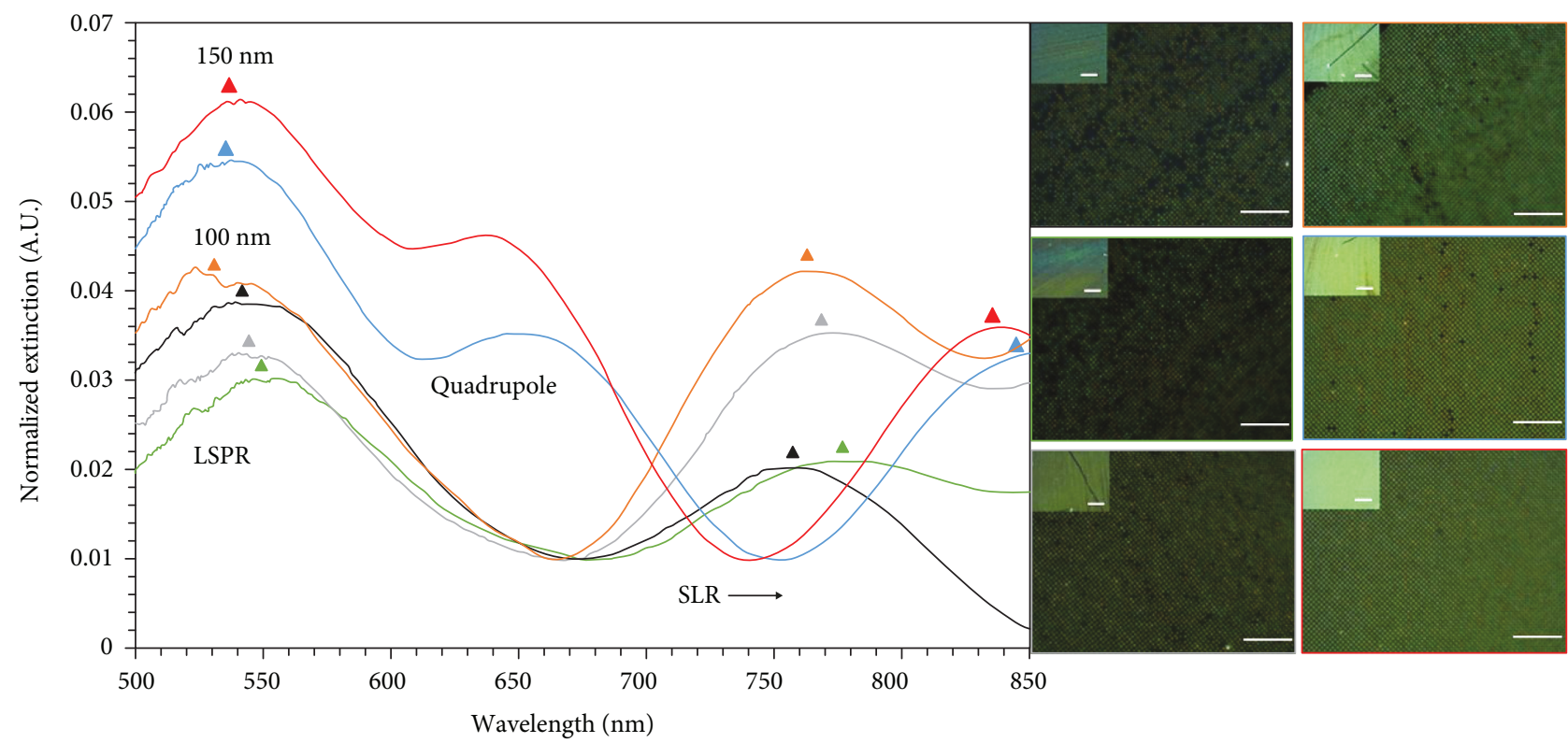

FIGURE 2: Extinction spectra for the six arrays shown in Figure 1 normalizing the valley before the SLR peak to 0.01 A.U. Colored triangles represent the peak locations for both LSPR and SLR features in spectra.

of the plot in Figure 2. LSPR and SLR peak locations are designated in Figure 2 via a filled triangle matching the line color of the normalized extinction spectra. Extinction spectra for $150 \mathrm{~nm}$ AuNPs differ from extinction spectra of $100 \mathrm{~nm}$ AuNPs in that the $150 \mathrm{~nm}$ AuNPs have a third feature arising from a quadrupole mode at $\sim 650 \mathrm{~nm}$ wavelength [39]. Highdensity ordered arrays like those shown in Figure 1 resulted in observable blueshifts in measured extinction spectra and extinction/NP calculations, which correspond to estimates of DeJarnette et al. previously reported $[5,13]$. The LSPR and SLR peak locations blueshift as the density increases (see Figure 2) for the green, gray, and orange lines. For the LSPR feature, the peak locations were $\sim 555,550$, and $535 \mathrm{~nm}$, and the locations for the SLR peaks were $\sim 778$, 773 , and $765 \mathrm{~nm}$ for the green, gray, and orange spectra, respectively. The spectra for the black line (LSPR $=547 \mathrm{~nm}$ and SLR $=756 \mathrm{~nm}$ ) does not follow this trend, which was likely due to multiple layers of particles rather than single layers of particles like the other three spectra. The SLR and quadrupole peaks for the $150 \mathrm{~nm}$ AuNP spectra also blueshifts as the density increases, but the LSPR peak has a slight redshift (538 nm for blue line and $541 \mathrm{~nm}$ for red line).

Matricardi et al. showed that as the lattice spacing increases, the peak height decreases [19]. This work maintained a lattice spacing of $600 \mathrm{~nm}$ and increased the density of filled cavities for $100 \mathrm{~nm}$ AuNP arrays resulting in observable increases in peak heights (estimated by subtracting a baseline of 0.01 A.U. from the extinction at the LSPR or SLR peak). The density for the image boxed in gray was significantly higher than the density for the image boxed in green, which resulted in a significant increase in the SLR peak height of 0.011 A.U. (green) to 0.025 A.U. (gray). This 2.3fold increase in peak height did not hold for the LSPR feature which had an increase from 0.020 A.U. (green) to 0.022 A.U. (gray) or a modest 1.1-fold increase. The difference in density between the gray and orange lines was minor but still resulted in 1.28- and 1.5-fold increases in peak height for the SLR and LSPR features, respectively.

LSPR and SLR peak locations were estimated for Mie theory, DDA, CDA, and rsa-CDA and compared to measured peak locations in Figure 3. Peak locations for Mie theory and DDA estimates $(590-595 \mathrm{~nm})$ were redshifted compared to measured values (530-565 nm) due to differences in the RI values. Since an exact material composition surrounding the AuNPs in the PDMS cavity was lacking, Mie theory and DDA assumed that the AuNPs were surrounded by a RI of PDMS (1.42), while the experimental system is surrounded by an unknown composition percentage of components of varying RI including air (1.00), Triton X-100 (1.49), water (1.33), and PDMS (1.42). Each material impacts the overall $\mathrm{RI}$ and either blueshifts (decreasing RI) or redshifts (increasing RI) the peak locations depending on which material surrounds the AuNPs at a higher fraction. CDA simulations estimated the LSPR peak location $(565 \mathrm{~nm})$ and the SLR peak location $(642 \mathrm{~nm})$ for $100 \mathrm{~nm}$ AuNPs where the LSPR peak showed a slight redshift and the SLR showed a significant blueshift compared to measured values ranging between 530-560 $\mathrm{nm}$ and $755-780 \mathrm{~nm}$, respectively. SLR peak location estimates were closer for $150 \mathrm{~nm}$ AuNPs compared to $100 \mathrm{~nm}$ AuNPs, which is likely due to the $150 \mathrm{~nm}$ AuNP occupying a larger volume of the cavity compared to the $100 \mathrm{~nm}$ AuNP. The diameter of $195 \mathrm{~nm}$ and a depth of $150 \mathrm{~nm}$ cavity dimensions also reduce the possibility of multiple $150 \mathrm{~nm}$ AuNPs occupying the same cavity. Peak locations for rsa-CDA simulations were closer to measured values than those of CDA for $150 \mathrm{~nm}$ AuNPs. CDA simulations assign an array size of $301 \times 301$ like rsa-CDA, but CDA uses a series of dipoles with a resolution of $5 \mathrm{~nm}$ to form each 100 and $150 \mathrm{~nm}$ AuNP while rsa-CDA assigns each AuNP as a single dipole point for faster computation of the results. 


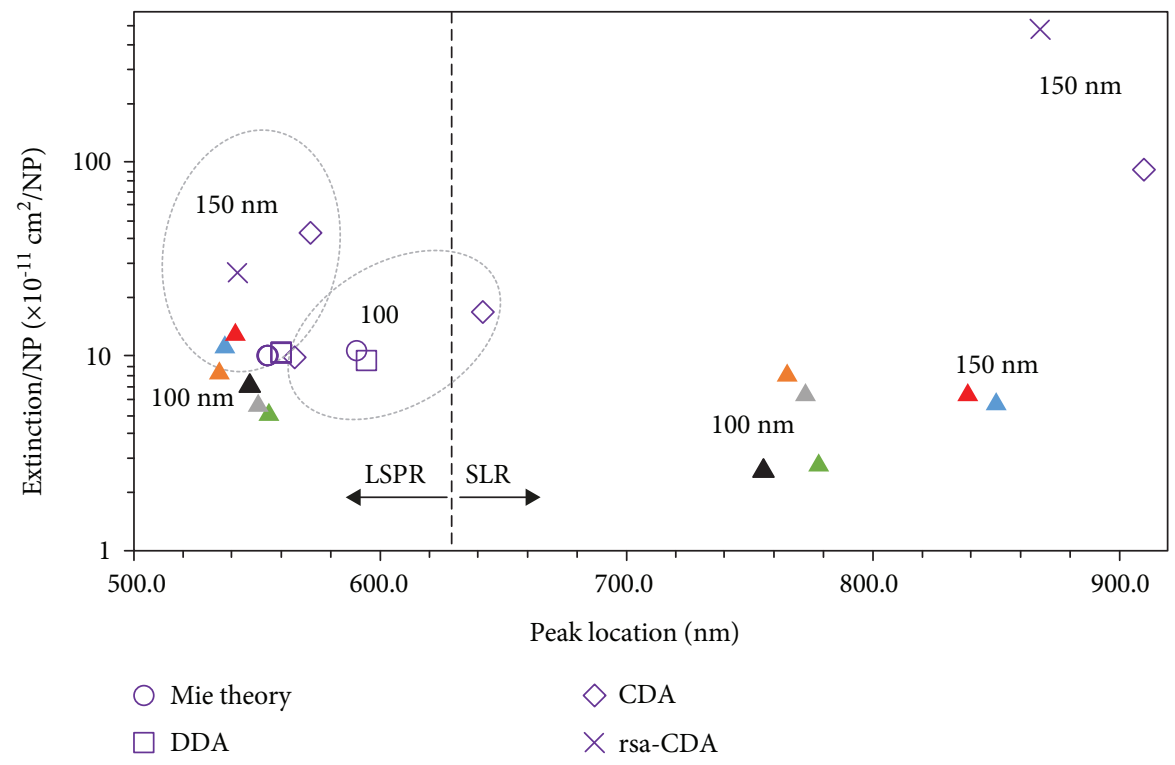

FIGURE 3: Extinction per NP calculations for measured (filled triangles with colors corresponding to spectra in Figure 2) and simulated (hollow symbols) data of 100 and $150 \mathrm{~nm}$ AuNP ordered arrays.

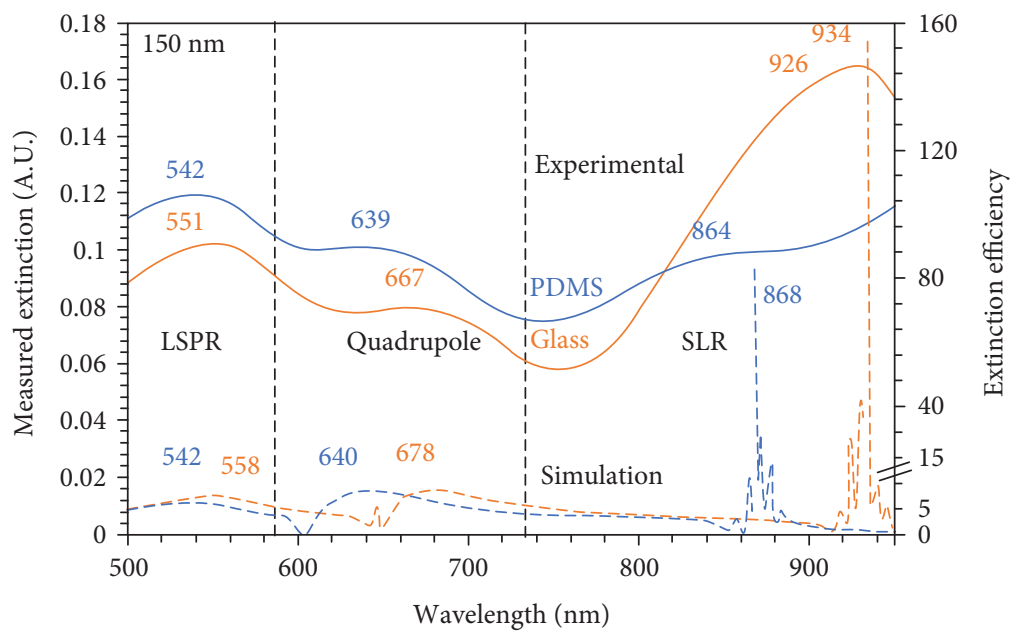

FIgURe 4: Measured extinction and rsa-CDA simulated extinction efficiency for an ordered array of $150 \mathrm{~nm}$ AuNPs in PDMS and transferred to glass.

Measured and estimated extinction/NP values for $150 \mathrm{~nm}$ AuNPs were higher than those for $100 \mathrm{~nm}$ AuNPs for LSPR peaks while the SLR peaks were redshifted $>50 \mathrm{~nm}$ for the $150 \mathrm{~nm}$ AuNPs compared to $100 \mathrm{~nm}$ AuNPs. Extinction/NP estimates for 100 and $150 \mathrm{~nm}$ AuNP LSPR peaks using single particle simulations (Mie theory and DDA) and array simulations (CDA and rsa-CDA) were compared to measured extinction/NP values calculated from the spectral peaks in Figure 2 (filled triangles), which is shown in Figure 3. Mie theory and DDA simulations estimated the extinction/NP $\left(9-10 \times 10^{-11}\right)$ for the $100 \mathrm{~nm}$ AuNPs and were compared to measured values ranging from $\left(5-8 \times 10^{-11}\right)$. The CDA simulations assigned a RI of PDMS (1.42) surrounding the entire AuNP, while the measured values were within $195 \mathrm{~nm}$ diameter PDMS cavities and were surrounded by water (1.33), Triton X-100 (1.49), and air (1.00) at varying fractions per cavity.

3.4. Experimental vs. Simulated Optical Properties. As discussed in Optical Properties of AuNP Ordered Arrays, the rsa-CDA simulations resulted in estimated peak locations comparable to measured peak locations for the $150 \mathrm{~nm}$ AuNP arrays and to previous estimated values reported by DeJarnette et al. [5, 13]. An ordered array containing $150 \mathrm{~nm}$ AuNPs was fabricated using the conditions shown in Figure 1 (red triangle, low evaporation rate, and $1.2 \mu \mathrm{m} / \mathrm{s}$ deposition rate). Optical spectra for this array were measured before the array was transferred to a glass slide via a process developed by Cerf and Vieu [26]. The spectral peak locations for rsa-CDA simulations in PDMS $(\mathrm{RI}=1.42)$ matched 
measured values for LSPR (542 vs. $542 \mathrm{~nm}$ ), quadrupole (640 vs. $639 \mathrm{~nm}$ ), and SLR ( 868 vs. $864 \mathrm{~nm}$ ) values within $0.46 \%$ as shown in Figure 4. After performing UV-Vis spectroscopy on the array in PDMS, the array was transferred onto a glass substrate where UV-Vis spectroscopy was again performed to characterize the arrays on glass after the transfer. The spectral peak locations for the simulation versus measured for LSPR (558 vs. $551 \mathrm{~nm}$ ), quadrupole (678 vs. $667 \mathrm{~nm}$ ), and SLR (934 vs. $926 \mathrm{~nm})$ on glass $(\mathrm{RI}=1.52)$ matched within $1.7 \%$. The $\sim 3.7$-fold increase in the percent difference between the PDMS and glass measured versus simulation data is likely due to the decrease from $\sim 100 \%$ density of filled cavities in PDMS to $\sim 85 \%$ density on glass.

Not only were the peak locations comparable between experimental and simulation spectra, but the relative increase in peak heights was comparable. The LSPR peak height appeared to slightly increase in the experimental results for glass ( 0.09 A.U.) compared to PDMS ( 0.04 A.U.). This relative doubling of the measured peak heights was also observed in the simulation results as the LSPR peak height for the simulation of the array on glass appears to be slightly above the simulation spectra for PDMS. This was also observed for the quadrupole peak in which the experimental and simulation results for both glass and PDMS was the same. The experimental peak height for the array on glass ( $\sim .1$ A.U.) was nearly double the experimental peak height for the array in PDMS ( 0.05 A.U.) while the simulation peak heights were also nearly double for the arrays on glass compared to the arrays in PDMS.

\section{Conclusions}

In summary, directed self-assembly was used to fabricate square arrays of AuNPs resulting in large-area arrays with observable optical enhancements as the density of filled cavities increased. Controlling the evaporation rate, deposition rate, and AuNP size resulted in $>2 \mathrm{~mm} \times 2 \mathrm{~mm}$ arrays with $\sim 100 \%$ filled cavities containing one AuNP per cavity. Extinction spectra showed that as the density of filled cavities increased, both the LSPR and SLR peak locations blueshifted and the peak heights increased. Extinction/NP calculations for measured 100 and $150 \mathrm{~nm}$ AuNPs in square arrays were also blueshifted compared to simulated values for single particles (Mie theory and DDA) and finite arrays (CDA and rsaCDA). The SLR peak height was shown to significantly increase when transferred from inside PDMS cavities onto a glass substrate. The experimental peak locations for the arrays both in PDMS and on glass were matched within 0.5 and $1.7 \%$, respectively, compared to rsa-CDA simulations.

\section{Data Availability}

The data used to support the findings of this study will be archived on the storage drive at Utah State University for at least three years beyond the end of the publication period. The storage drive has over $50 \mathrm{~Tb}$ of available disk space for this purpose. This drive is reliably backed up (both daily snapshots and weekly full backups) to prevent data loss.
The corresponding author will share project data in accordance with the NSF Data Management Plan requirements.

\section{Conflicts of Interest}

The authors declare no competing financial interest.

\section{Authors' Contributions}

K. Berry refined the directed self-assembly process that yielded reproducible, large-area, and high-density ordered arrays and drafted text and figures. R. Romo performed the simulations and added text discussing the simulations. $\mathrm{M}$. Mitchell assisted in drafting the table of self-assembly references, added text discussing the table, and fabricated blank stamps used for self-assembly experiments. V. Bejugam built the cage and fabricated blank stamps. D. K. Roper directed the work and refined text and figures.

\section{Acknowledgments}

This research work was supported by the Center for Advanced Surface Engineering (CASE), under the National Science Foundation (NSF) Grant No. OIA-1457888 and the Arkansas EPSCoR Program. Dr. Roper also acknowledges support from Utah State University. The authors would like to thank Megan Lanier for fabricating additional blank stamps and Loc Huynh for performing preliminary self-assembly experiments.

\section{References}

[1] V. Amendola, R. Pilot, M. Frasconi, O. M. Maragò, and M. A. Iatì, "Surface plasmon resonance in gold nanoparticles: a review," Journal of Physics: Condensed Matter, vol. 29, no. 20, article 203002, 2017.

[2] J. R. Dunklin, C. Bodinger, G. T. Forcherio, and D. Keith Roper, "Plasmonic extinction in gold nanoparticle-polymer films as film thickness and nanoparticle separation decrease below resonant wavelength," Journal of Nanophotonics, vol. 11, no. 1, article 016002, 2017.

[3] V. G. Kravets, A. V. Kabashin, W. L. Barnes, and A. N. Grigorenko, "Plasmonic surface lattice resonances: a review of properties and applications," Chemical Reviews, vol. 118, no. 12 , pp. 5912-5951, 2018.

[4] V. Bejugam, "Opto-thermal characterization of plasmon and coupled lattice resonances in 2-D metamaterial arrays," Theses and Dissertations, vol. 2868, 2018, https://scholarworks.uark .edu/etd/2868.

[5] D. DeJarnette, J. Norman, and D. K. Roper, "Attribution of Fano resonant features to plasmonic particle size, lattice constant, and dielectric wavenumber in square nanoparticle lattices," Photonics Research, vol. 2, no. 1, 2014.

[6] G. T. Forcherio, P. Blake, M. Seeram, D. DeJarnette, and D. K. Roper, "Coupled dipole plasmonics of nanoantennas in discontinuous, complex dielectric environments," Journal of Quantitative Spectroscopy and Radiative Transfer, vol. 166, pp. 93-101, 2015.

[7] Y. Francescato, V. Giannini, and S. A. Maier, "Plasmonic systems unveiled by Fano resonances," ACS Nano, vol. 6, no. 2, pp. 1830-1838, 2012. 
[8] R. Guo, T. K. Hakala, and P. Törmä, "Geometry dependence of surface lattice resonances in plasmonic nanoparticle arrays," Physical Review B, vol. 95, no. 15, pp. 1-11, 2017.

[9] A. D. Humphrey and W. L. Barnes, "Plasmonic surface lattice resonances on arrays of different lattice symmetry," Physical Review B, vol. 90, no. 7, pp. 1-8, 2014.

[10] A. I. Väkeväinen, R. J. Moerland, H. T. Rekola et al., "Plasmonic surface lattice resonances at the strong coupling regime," Nano Letters, vol. 14, no. 4, pp. 1721-1727, 2014.

[11] B. Auguié, X. M. Bendaña, W. L. Barnes, and F. J. García de Abajo, "Diffractive arrays of gold nanoparticles near an interface: critical role of the substrate," Physical Review B, vol. 82, no. 15, article 155447, 2010.

[12] P. Blake, S. Kühne, G. T. Forcherio, and D. K. Roper, "Diffraction in nanoparticle lattices increases sensitivity of localized surface plasmon resonance to refractive index changes," Journal of Nanophotonics, vol. 8, no. 1, 2014.

[13] D. DeJarnette, G. G. Jang, P. Blake, and D. K. Roper, "Polarization angle affects energy of plasmonic features in Fano resonant regular lattices," Journal of Optics, vol. 16, no. 10, article 105006, 2014.

[14] U. Fano, "Effects of configuration interaction on intensities and phase shifts," Physics Review, vol. 124, no. 6, pp. 18661878, 1961.

[15] N. Flidj, G. Laurent, J. Aubard et al., "Grating-induced plasmon mode in gold nanoparticle arrays," The Journal of Chemical Physics, vol. 123, no. 22, article 221103, 2005.

[16] G. T. Forcherio, P. Blake, D. DeJarnette, and D. K. Roper, "Nanoring structure, spacing, and local dielectric sensitivity for plasmonic resonances in Fano resonant square lattices," Optics Express, vol. 22, no. 15, pp. 17791-17803, 2014.

[17] S. Baur, S. Sanders, and A. Manjavacas, "Hybridization of lattice resonances," ACS Nano, vol. 12, no. 2, pp. 1618-1629, 2018.

[18] D. Khlopin, F. Laux, W. P. Wardley et al., "Lattice modes and plasmonic linewidth engineering in gold and aluminum nanoparticle arrays," Journal of the Optical Society of America B: Optical Physics, vol. 34, no. 3, p. 691, 2017.

[19] C. Matricardi, C. Hanske, J. L. Garcia-Pomar, J. Langer, A. Mihi, and L. M. Liz-Marzán, "Gold nanoparticle plasmonic superlattices as surface enhanced Raman spectroscopy substrates," ACS Nano, vol. 12, no. 8, pp. 8531-8539, 2018.

[20] P. Blake, J. Obermann, B. Harbin, and D. K. Roper, "Enhanced nanoparticle response from coupled dipole excitation for plasmon sensors," IEEE Sensors Journal, vol. 11, no. 12, pp. 33323340, 2011.

[21] V. Komanicky, H. Iddir, K. C. Chang et al., "Shape-dependent activity of platinum array catalyst," Journal of the American Chemical Society, vol. 131, no. 16, pp. 5732-5733, 2009.

[22] T. Mårtensson, P. Carlberg, M. Borgström, L. Montelius, W. Seifert, and L. Samuelson, "Nanowire arrays defined by nanoimprint lithography," Nano Letters, vol. 4, no. 4, pp. 699702, 2004.

[23] P. Nagpal, N. C. Lindquist, S. H. Oh, and D. J. Norris, "Ultrasmooth patterned metals for plasmonics and metamaterials," Science, vol. 325, no. 5940, pp. 594-597, 2009.

[24] M. Tajdidzadeh, A. B. Zakaria, Z. A. Talib, A. S. Gene, and S. Shirzadi, "Optical nonlinear properties of gold nanoparticles synthesized by laser ablation in polymer solution," Journal of Nanomaterials, vol. 2017, Article ID 4803843, 9 pages, 2017.
[25] W. Ahn and D. K. Roper, "Periodic nanotemplating by selective deposition of electroless gold island films on particlelithographed dimethyldichlorosilane layers," ACS Nano, vol. 4, no. 7, pp. 4181-4189, 2010.

[26] A. Cerf and C. Vieu, "Transfer printing of sub-100 nm nanoparticles by soft lithography with solvent mediation," Colloids and Surfaces A: Physicochemical and Engineering Aspects, vol. 342, no. 1-3, pp. 136-140, 2009.

[27] J. A. Fan, K. Bao, L. Sun et al., "Plasmonic mode engineering with templated self-assembled nanoclusters," Nano Letters, vol. 12, no. 10, pp. 5318-5324, 2012.

[28] F. Juillerat, H. H. Solak, P. Bowen, and H. Hofmann, "Fabrication of large-area ordered arrays of nanoparticles on patterned substrates," Nanotechnology, vol. 16, no. 8, pp. 1311-1316, 2005.

[29] C. Kinnear, J. Cadusch, H. Zhang et al., "Directed chemical assembly of single and clustered nanoparticles with silanized templates," Langmuir, vol. 34, no. 25, pp. 7355-7363, 2018.

[30] D. E. Lee, J. Ryu, D. Hong, S. Park, D. H. Lee, and T. P. Russell, "Directed self-assembly of asymmetric block copolymers in thin films driven by uniaxially aligned topographic patterns," ACS Nano, vol. 12, no. 2, pp. 1642-1649, 2018.

[31] L. Malaquin, T. Kraus, H. Schmid, E. Delamarche, and H. Wolf, "Controlled particle placement through convective and capillary assembly," Langmuir, vol. 23, no. 23, pp. 11513$11521,2007$.

[32] Y. Yin, Y. Lu, B. Gates, and Y. Xia, “Template-assisted selfassembly: a practical route to complex aggregates of monodispersed colloids with well-defined sizes, shapes, and structures," Journal of the American Chemical Society, vol. 123, no. 36, pp. 8718-8729, 2001.

[33] H. Zhang, J. Cadusch, C. Kinnear, T. James, A. Roberts, and P. Mulvaney, "Direct assembly of large area nanoparticle arrays," ACS Nano, vol. 12, no. 8, pp. 7529-7537, 2018.

[34] N. A. A. Mohd Arif, C. C. Jiun, and S. Shaari, "Effect of annealing temperature and spin coating speed on Mn-doped $\mathrm{ZnS}$ nanocrystals thin film by spin coating," Journal of Nanomaterials, vol. 2017, Article ID 2560436, 6 pages, 2017.

[35] E. K. Wujcik, S. R. Aceto, R. Narayanan, and A. Bose, "Lead selenide nanostructures self-assembled across multiple length scales and dimensions," Journal of Nanomaterials, vol. 2016, Article ID 9575839, 6 pages, 2016.

[36] D. Dejarnette, P. Blake, G. T. Forcherio, and D. Keith Roper, "Far-field Fano resonance in nanoring lattices modeled from extracted, point dipole polarizability," Journal of Applied Physics, vol. 115, no. 2, article 024306, 2014.

[37] D. Dejarnette and D. K. Roper, "Electron energy loss spectroscopy of gold nanoparticles on graphene," Journal of Applied Physics, vol. 116, no. 5, article 054313, 2014.

[38] M. Seeram, G. T. Forcherio, and D. K. Roper, Shape Generator for the DDSCAT Software, nanoHUB, 2016.

[39] C. P. Burrows and W. L. Barnes, "Large spectral extinction due to overlap of dipolar and quadrupolar plasmonic modes of metallic nanoparticles in arrays," Optics Express, vol. 18, no. 3, pp. 3187-3198, 2010. 


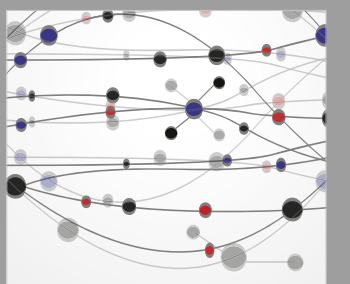

The Scientific World Journal
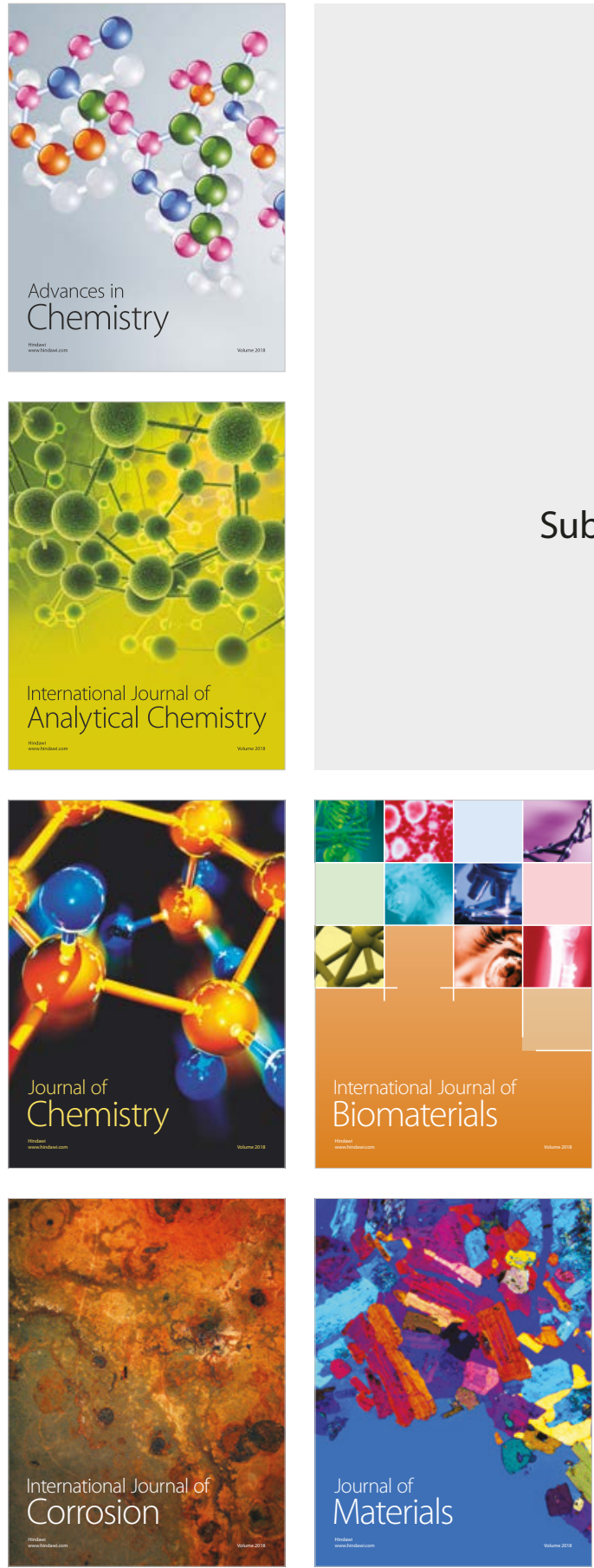

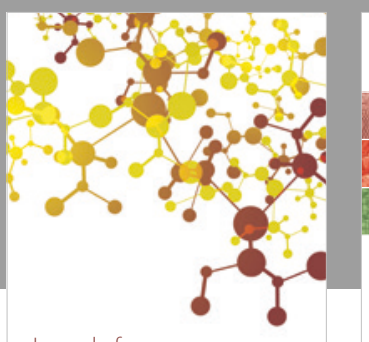

Journal of

Applied Chemistry
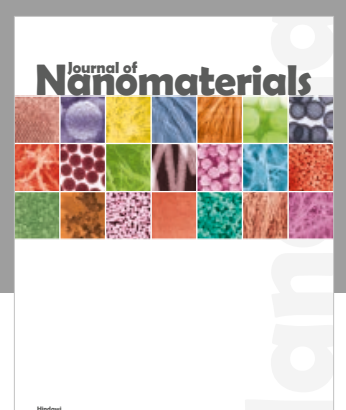

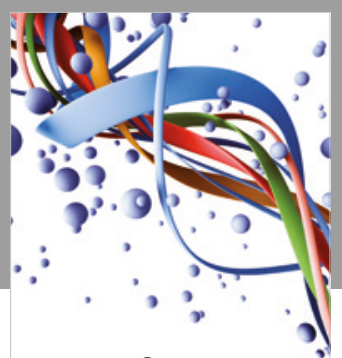

Scientifica

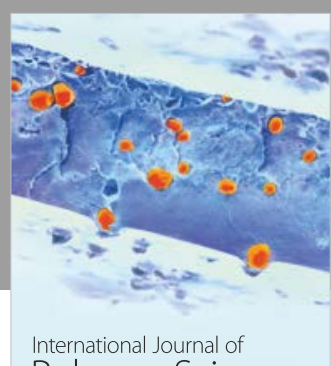

Polymer Science

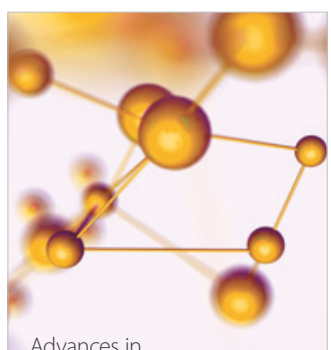

Physical Chemistry
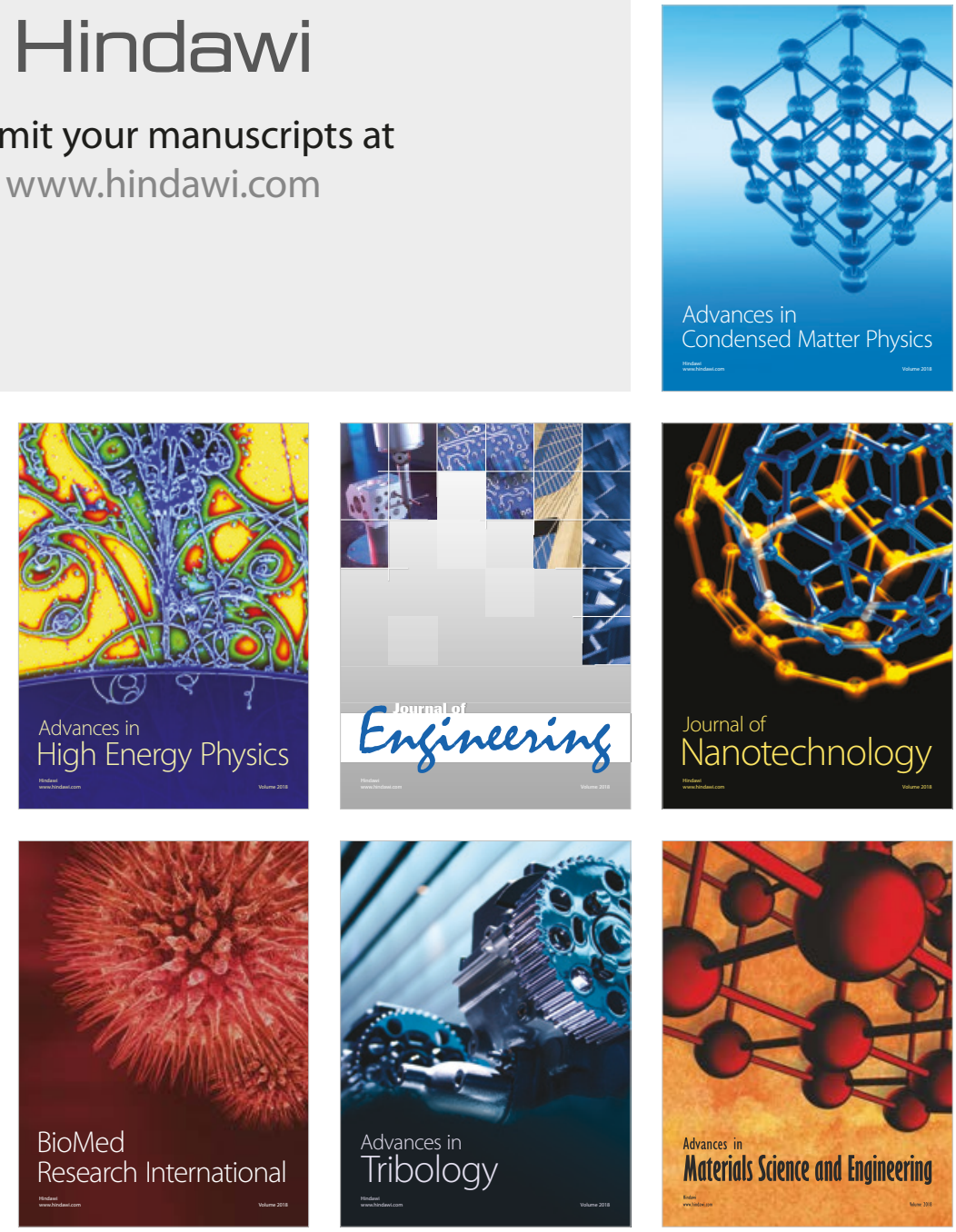